\title{
Signature candidate of quantum chaos far from the semiclassical regime
}

\author{
Shang-Bin Li and Zhengyuan Xu \\ School of Information Science and Technology, and Optical Wireless Communication and Network Center, \\ University of Science and Technology of China, Hefei, Anhui 230027, P.R. China
}

\begin{abstract}
We numerically investigated the entanglement product in the simplest coupled kicked top model with the spin $j=1$. Different from the dynamical pattern of entanglement in the semiclassical regime, two similar initial states may have discordant entanglement oscillations. A candidate of the quantum signature of this classical chaotic system was proposed. The potential antimonotonic relation between the rank correlation coefficient qualifying the concordant of two entanglement evolutions and the stationary entanglement was preliminarily revealed.
\end{abstract}

PACS numbers: 05.45.Mt, 03.65.Ud, 03.67.-a

The distinct dynamical behaviors of entanglement in the simplest coupled kicked top model with the $\operatorname{spin} j=1$ were found. It was shown two similar initial states may have discordant entanglement oscillations. Based on it, the rank correlation coefficient qualifying the concordant of two entanglement evolutions was proposed as the candidate of the quantum signature of this classical chaotic system, which has the potential antimonotonic relation with the stationary entanglement caused by a similar dynamical process as quantum quench.

Entanglement in the quantum chaotic systems has been studied by several authors [1 24]. For the system of weak coupled kicked tops, in the semiclassical regime, it has been clarified that two initially separable subsystems can get entangled in a nearly linear rate depending on the intrinsic chaotic properties, and their entanglement eventually reaches saturation $[8-11,20]$. It has also been elucidated that the increment of the nonlinear parameter of weakly coupled kicked tops does not accelerate the entanglement production in the strongly chaotic region [11]. For strong coupled kicked tops in the semiclassical regime, it is observed the greater the kick strength, the higher asymptotic value of entanglement if the kick strength is not very small [10, 20]. In the deep quantum regime, based on the combined electronic and nuclear spin of a single atom, the experimental realization of the quantum kicked top with $j=3$ has been presented and good correspondence between the quantum dynamics and classical phase space structures has been found [3]. Clear differences in the sensitivity to perturbation in chaotic versus regular regimes, and experimental evidence for dynamical entanglement as a signature of chaos have also been observed [3]. In Ref.[25], it has been shown that the discrepancy between quantum and classical evolutions can be significantly decreased by even a weak loss of the coherence. Motivated by previous works, we investigated the entanglement quantified by log-negativity in coupled kicked tops with the spin $j=1$ by using $\rho=p\left|\psi_{1}\right\rangle\left\langle\psi_{1}|\otimes| \psi_{2}\right\rangle\left\langle\psi_{2}|+(1-p)| \psi_{2}\right\rangle\left\langle\psi_{2}\right| \otimes$ $\left|\psi_{1}\right\rangle\left\langle\psi_{1}\right|$ as the initial state. The difference of the VonNeumann entropy between $\rho_{p=0}$ and $\rho_{p=0.5}$ is given by
$\Delta S=1-\frac{1}{\ln 4}\left[\left(1-\left|\left\langle\psi_{1} \mid \psi_{2}\right\rangle\right|^{2}\right) \ln \left(1-\left|\left\langle\psi_{1} \mid \psi_{2}\right\rangle\right|^{2}\right)+(1+\right.$ $\left.\left.\left|\left\langle\psi_{1} \mid \psi_{2}\right\rangle\right|^{2}\right) \ln \left(1+\left|\left\langle\psi_{1} \mid \psi_{2}\right\rangle\right|^{2}\right)\right]$, which depends on the overlap of $\left|\psi_{1}\right\rangle$ and $\left|\psi_{2}\right\rangle$ and $\Delta S \in[0,1]$. The purpose of choosing this kind of initial states is to keep the energy independent on the initial mixedness for the following coupled symmetric kicked tops. Moreover, this kind of initial states guarantees the mixedness-independent dynamical trajectories of arbitrary symmetric collective observables such as $\hat{J}_{z_{1}}^{2}+\hat{J}_{z_{2}}^{2}$ or $\hat{J}_{z_{1}} \hat{J}_{z_{2}}$ in two coupled symmetric tops. It is rationally conjectured that, in most cases, the characteristics of the entanglement evolution $E_{2}(t)$ corresponding to the above initial state with $p=0.5$ reflect closer details concerning its classical counterpart than the entanglement evolution $E_{1}(t)$ with initial $p=0$. Since in most of the processes of the quantum-classical transition, it comes with the loss or gain of the information 11. Good quantum-classical corresponding reflects the robustness of the system to the change of the information entropy [1]. Using the above specific initial states to elucidate the sensitivity of the dynamics to the initial change of no more than 1-qubit information in this simplest coupled kicked tops can help us to understand the quantum chaos in deep quantum regime. This is our main motivation. In order to investigate how the initially lost information affects the entanglement dynamical behaviors of two tops with small spin, it is proposed to apply the scaled rank correlation coefficient 26]

$\eta_{d} \equiv \lim _{N \rightarrow \infty} \frac{|\Delta S|}{N} \sum_{t=1}^{N}\left[\Theta\left(E_{1}(t)-E_{1}(t-1)\right) \Theta\left(E_{2}(t)-E_{2}(t-1)\right)\right]$

to quantify the concordant or discordant between two entanglement evolution, where $\Theta(x>0)=1, \Theta(x=0)=0$ and $\Theta(x<0)=-1$. The $\eta_{d}$ closer to 1 indicates two entanglement evolutions are more concordant. For the weak coupled kicked tops with $j>50$, previous studies have shown the entanglement between two tops does not exhibit large amplitude oscillation but monotonic ascent to saturation [8 11, 20]. In these cases, $\eta_{d}$ is always near one which can not provide the explicit quantum signature of its classical chaotic counterpart. A cross correlation coefficient $\eta_{g}$ is also defined for comparing with $\eta_{d}$ 

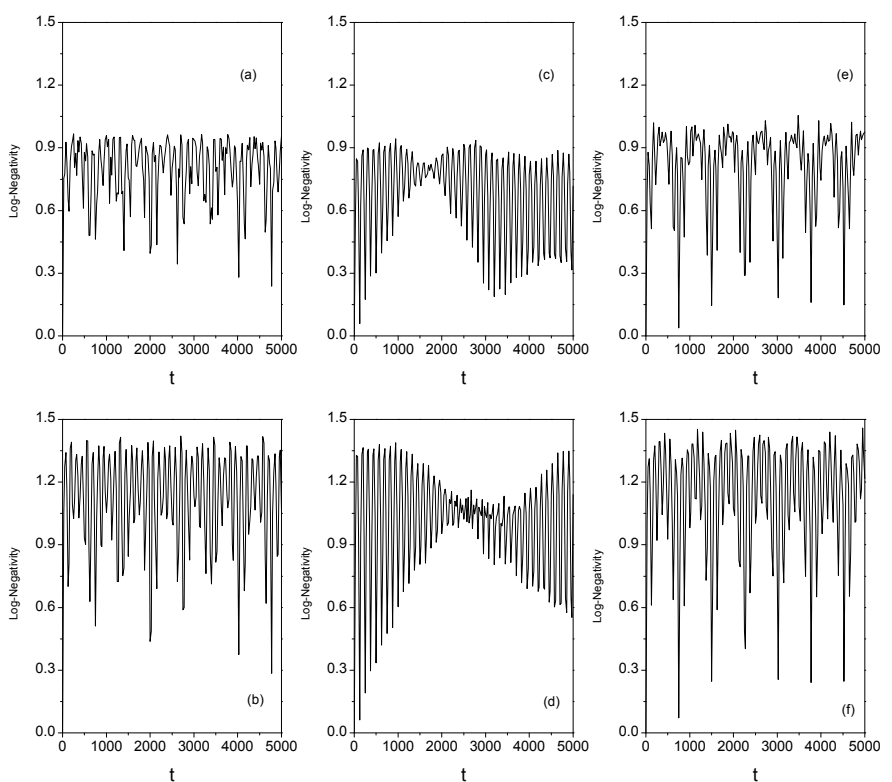

FIG. 1: The log-negativity of two tops is plotted as the function of $t$ for three different nonlinear coefficients and for pure or mixed spin coherent state. $j=1 ; \varepsilon=0.05 ; \gamma_{1}=-3$; $\gamma_{2}=3$; (a) $k=0.25, p=0.5$; (b) $k=0.25, p=0.0$; (c) $k=3$, $p=0.5$; (d) $k=3, p=0.0$; (e) $k=6, p=0.5$; (f) $k=6$, $p=0.0$.
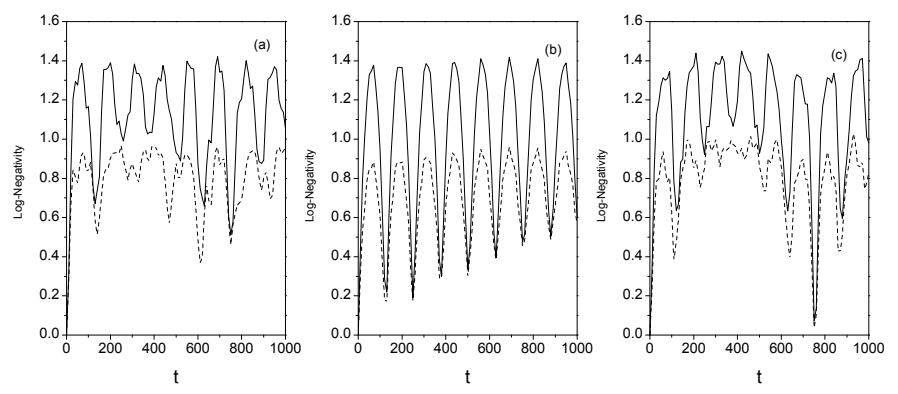

FIG. 2: The same parameters as Fig.1; (a) $k=0.25$; (b) $k=3$; (c) $k=6$; (Solid line) $p=0$; (Dash line) $p=0.5$. The scaled rank correlation coefficients $\eta_{d}$ corresponding to $k$ have the order relation $\eta_{d}(k=0.25)<\eta_{d}(k=6)<\eta_{d}(k=3)$. For other initial state with $\gamma_{1}=-\gamma_{2}=1$ (a fixed point in phase space of the single kicked top with $k=3$ in Ref.[8]), we have also calculated the parameter $\eta_{d}$ and obtained $\eta_{d}(k=0.25)=$ $0.314<\eta_{d}(k=6)=0.410<\eta_{d}(k=3)=0.448$.

as follows:

$\eta_{g} \equiv \lim _{N \rightarrow \infty} \frac{1}{N} \sum_{t=1}^{N}\left[\left(E_{1}(t)-E_{1}(t-1)\right)\left(E_{2}(t)-E_{2}(t-1)\right)\right]$,

which may also characterize the synchronization of the time variations of $E_{1}(t)$ and $E_{2}(t)$.

The Hamiltonian of the coupled kicked tops can be written as [8]:

$$
\mathcal{H}(t)=\hat{H}_{1}(t)+\hat{H}_{2}(t)+\hat{H}_{12}(t)
$$
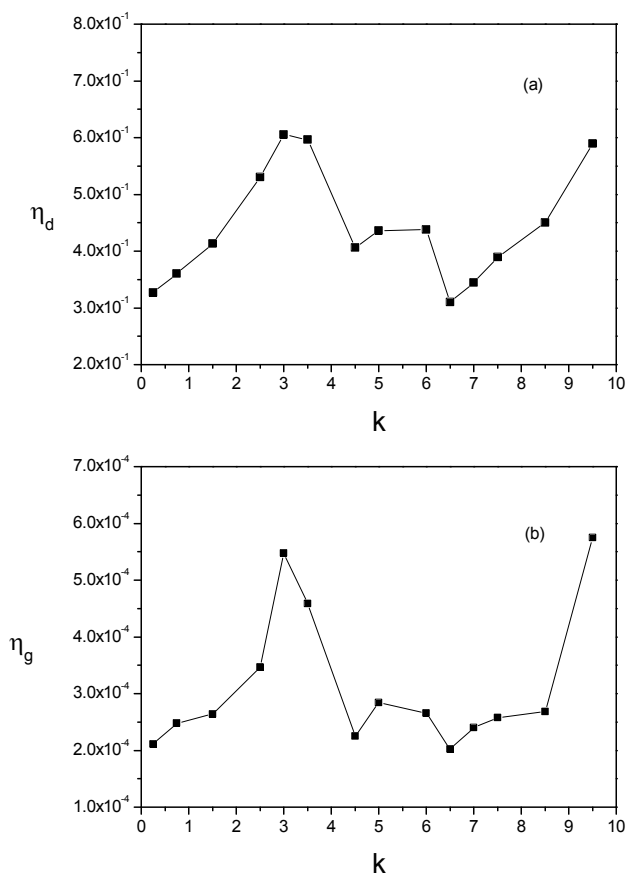

FIG. 3: The correlation coefficients (a) $\eta_{d}$ and (b) $\eta_{g}$ are plotted as the function of $k . j=1 ; \varepsilon=0.05 ; \gamma_{1}=-3$; $\gamma_{2}=3$.

$$
\begin{aligned}
\text { with } \hat{H}_{i}(t) & \equiv \frac{\pi}{2} \hat{J}_{y_{i}}+\frac{k_{i}}{2 j} \hat{J}_{z_{i}}^{2} \sum_{n} \delta(t-n), \\
\hat{H}_{12}(t) & \equiv \frac{\varepsilon}{j} \hat{J}_{z_{1}} \hat{J}_{z_{2}} \sum_{n} \delta(t-n),
\end{aligned}
$$

where $i=1,2$, and $\hat{H}_{i}(t)$ 's are the Hamiltonians of the individual tops. $\hat{H}_{12}(t)$ represents the coupling between two tops with a coupling strength of $\varepsilon / j$. All these spin operators obey standard commutation relations. Choosing the simultaneous eigenstates $\left|j_{1}, m_{1}\right\rangle_{1} \otimes\left|j_{2}, m_{2}\right\rangle_{2}$ of the four mutually commuting operators $\hat{J}_{i}^{2}$ and $\hat{J}_{z_{i}}(i=$ $1,2)$ as the basis, where $\hat{J}_{i}^{2}\left|j_{i}, m_{i}\right\rangle_{i}=j_{i}\left(j_{i}+1\right)\left|j_{i}, m_{i}\right\rangle_{i}$ and $\hat{J}_{z_{i}}\left|j_{i}, m_{i}\right\rangle_{i}=m_{i}\left|j_{i}, m_{i}\right\rangle_{i}$. Thereafter, it is assumed $j_{1}=j_{2}=j$ and $k_{1}=k_{2}=k$ for the coupled kicked symmetrical tops.

In previous studies, usually the pure direct product state of two spin coherent states [8 11, 20] is chosen as the initial state of the total system. Here, we use a mixed state of direct product of two spin coherent states as our initial state for two tops. The spin coherent state is given in $\left|j, m_{i}\right\rangle_{i}$ basis as :

$$
|\gamma\rangle_{i}=\left(1+|\gamma|^{2}\right)^{-j} \sum_{m_{i}=-j}^{j} \gamma^{j-m_{i}} \sqrt{\left(\begin{array}{c}
2 j \\
j+m_{i}
\end{array}\right)}\left|j, m_{i}\right\rangle_{i}
$$

where $\gamma \equiv \exp (i \phi) \tan (\theta / 2)$. The initial normalized mixed state can be written as

$$
\rho(0)=p\left|\psi^{+}(0)\right\rangle\left\langle\psi^{+}(0)|+(1-p)| \psi^{-}(0)\right\rangle\left\langle\psi^{-}(0)\right|,
$$


where $\left|\psi^{+}(0)\right\rangle$ and $\left|\psi^{-}(0)\right\rangle$ are given by:

$$
\begin{aligned}
\left|\psi^{+}(0)\right\rangle & =\left|\gamma_{1}\right\rangle_{1}\left|\gamma_{2}\right\rangle_{2} \\
\left|\psi^{-}(0)\right\rangle & =\left|\gamma_{2}\right\rangle_{1}\left|\gamma_{1}\right\rangle_{2},
\end{aligned}
$$

and $p \in[0,1]$ is the real number.

The log-negativity of a bipartite density matrix is defined by [27]

$$
\mathcal{N}(\rho)=\log _{2}\left\|\rho^{\Gamma}\right\|,
$$

where $\rho^{\Gamma}$ is the partial transpose of $\rho$ and $\left\|\rho^{\Gamma}\right\|$ denotes the trace norm of $\rho^{\Gamma}$, which is the sum of the singular values of $\rho^{\Gamma}$. The coupled kicked tops with $j=1$ is far from the semiclassical regime. Due to the exchange symmetry of $\mathcal{H}(t)$ and its Floquet operator $\hat{U}$, the Floquet eigenstates have well-defined exchange parity: $j_{e}=(j+1)(2 j+1)$ Floquet eigenstates $\left|e_{i}\right\rangle$ with even exchange parity and $j_{o}=j(2 j+1)$ Floquet eigenstates $\left|o_{i}\right\rangle$ with odd exchange parity. The Floquet operator $\hat{U}$ can be expressed as

$$
\hat{U}=\sum_{i=1}^{j_{e}} e^{\imath \phi_{i}}\left|e_{i}\right\rangle\left\langle e_{i}\left|+\sum_{i=j_{e}+1}^{j_{e}+j_{o}} e^{\imath \phi_{i}}\right| o_{i}\right\rangle\left\langle o_{i}\right| .
$$

The initial state can be expanded by the Floquet states as

$$
\left|\gamma_{2}\right\rangle_{1}\left|\gamma_{1}\right\rangle_{2}=\sum_{i=1}^{j_{e}} a_{i}\left|e_{i}\right\rangle+\sum_{i=j_{e}+1}^{j_{e}+j_{o}} b_{i}\left|o_{i}\right\rangle
$$

and

$$
\left|\gamma_{1}\right\rangle_{1}\left|\gamma_{2}\right\rangle_{2}=\sum_{i=1}^{j_{e}} a_{i}\left|e_{i}\right\rangle-\sum_{i=j_{e}+1}^{j_{e}+j_{o}} b_{i}\left|o_{i}\right\rangle
$$

where $a_{i}$ and $b_{i}$ are the complex coefficients. The evolving state corresponding to the initial states $\rho_{p=0}(0)$ and $\rho_{p=0.5}(0)$ can be obtained as

$$
\hat{U}^{n}\left|\gamma_{2}\right\rangle_{1}\left|\gamma_{1}\right\rangle_{2}=\sum_{i=1}^{j_{e}} a_{i} e^{\imath n \phi_{i}}\left|e_{i}\right\rangle+\sum_{i=j_{e}+1}^{j_{e}+j_{o}} b_{i} e^{\imath n \phi_{i}}\left|o_{i}\right\rangle,
$$

and

$$
\begin{aligned}
\hat{U}^{n} \rho_{p=0.5}(0) \hat{U}^{\dagger n} & =\sum_{i, l=1}^{j_{e}} a_{i} a_{l}^{*} e^{\imath n\left(\phi_{i}-\phi_{l}\right)}\left|e_{i}\right\rangle\left\langle e_{l}\right| \\
& +\sum_{i, l=j_{e}+1}^{j_{e}+j_{o}} b_{i} b_{l}^{*} e^{\imath n\left(\phi_{i}-\phi_{l}\right)}\left|o_{i}\right\rangle\left\langle o_{l}\right|,
\end{aligned}
$$

respectively. Comparing the above two evolving states, it is explicit that the scaled rank correlation coefficient $\eta_{d}$ reflects the influence of coherent superposition between two Hilbert subspaces with different exchange parity on the dynamical behaviors of entanglement. In Figs.(1-2), we can see the log-negativity of the time evolution density operator for two different mixedness and for $k=6$, $k=3$ and $k=0.25$, which are strong chaotic, weak chaotic and regular cases of the single kicked top in previous literatures [28], respectively. For all of the cases, we pick an initial state in Eqs.(7-8) with $\gamma_{1}=-3$ and $\gamma_{2}=3$. Slightly different from the results in Ref.[3], the log-negativity shows quasi-periodic behavior and collapses and revivals of the oscillation for $k=3$. While for the case of $k=6$, the global dynamical pattern of the lognegativity is quasi-periodic but local dynamical pattern is chaotic. For small value of $k=0.25$, i.e. the regular case, the log-negativity only exhibits a chaotic oscillation. For the mixed initial state in Eqs.(7-8), the evolving lognegativity with $p=0.5$ is the lower bound of the one with $p=0$. The scaled rank correlation coefficient $\eta_{d}$ quantifying the concordant or discordant of the entanglement evolutions corresponding to initial states with $p=0$ and $p=0.5$ depends on $k$ even though the corresponding evolving states contain the same dynamics for arbitrary symmetric collective operators such as $\hat{J}_{z_{1}}^{2}+\hat{J}_{z_{2}}^{2}$ or the coupling term $\hat{J}_{z_{1}} \hat{J}_{z_{2}}$. From Fig.2(b), it can be found that the corresponding entanglement dynamical behaviors of two cases with $p=0$ and $p=0.5$ are synchronous and ordered when $k=3$, not like the disorder and asychronous correspondence in Fig.2(a) and Fig.2(c). In Fig.3, we plot $\eta_{d}$ and $\eta_{g}$ as the functions of $k$. It is shown both $\eta_{d}$ and $\eta_{g}$ firstly increase from very small value of $k$ and achieve their local maximal values near $k=3$, then nonmonotonically decrease to their local minimal values near $k=6.6$, and subsequently revival. For relating the scaled rank correlation coefficient $\eta_{d}$ in coupled kicked tops with $j=1, k=3$ and $\varepsilon=0.05$ with the previous analysis about its chaotic behaviors of classical counterpart, it is desirable to discuss the initial states dependence of $\eta_{d}$. For example, choosing four cases with $\gamma_{1}=-\gamma_{2}=$ $1, \tan \left(\frac{2.25}{2}\right) e^{0.63 i}, 3, \tan \left(\frac{0.89}{2}\right) e^{0.63 i}$ (fixed, fixed, chaotic, chaotic points in phase space of the single kicked top with $k=3$ in Ref. [8], respectively), the corresponding scaled rank correlation coefficients $\eta_{d}$ could be calculated and the results are $\eta_{d}=0.448,0.468,0.605,0.504$, respectively, which implies the more classical chaotic initial state of individual top will induce the more synchronous dynamical behaviors of $E_{1}(t)$ and $E_{2}(t)$.

In Ref. [3] , it has been experimentally demonstrated that less average entanglement generation for initial states localized in regular regions compared to those in the chaotic sea. Similarly, taking into account of the external pulse monitoring the collective observable $\hat{J}_{z_{1}}^{2}+\hat{J}_{z_{2}}^{2}$ of two spins which makes the states of two spins collapse toward the subspace with ${ }_{i}\left\langle\hat{J}_{z_{i}}^{2}\right\rangle_{i}=j^{2} \quad(i=1,2$, and ${ }_{i}\langle\hat{Y}\rangle_{i}$ denotes the expectation value of the operator $\hat{Y})$, we investigated the entanglement production in the coupled nonhermitian kicked tops by adding a positive imaginary part of $k$ in the Hamiltonian in Eq.(4). The results are shown in Figs.(4-6). Both the log-negativity of the tops with $k=0.25$ and $k=6$ exhibit the damped chaotic oscillation and converge to be stationary. The 
log-negativity at $t=1000$ firstly decreases with the increase of $\operatorname{Re}(k)$ from 0 to 3 , and reaches a flat bottom, then increases again and achieves a local maximal value near 6.4. For further increase of $\operatorname{Re}(k)$, it was numerically verified similar cycles repeat, and the stationary log-negativity is about 0.14 at $k=9$, where the stationary log-negativity is defined as $\mathcal{N}(t \rightarrow \infty)$. It has been verified the stationary log-negativity is independent of the initial pure or mixed states defined by Eqs.(7-8). From Fig.6(a), in the case with $\operatorname{Re}(k)=3$, it can be seen that the stationary log-negativity approximately linearly increases with the coupling strength $\varepsilon$ and the influence of $\operatorname{Im}(k)$ is negligible when $\varepsilon \leq 0.1$. Near $\varepsilon=1$, the stationary log-negativity achieves its local maximal values. Surprisingly, the stationary log-negativity could approach to 1.55, which implies, independent of the initial pure or mixed states, the stationary state is very close to the maximally entangled qutrits in the simplest nonHermitian coupled kicked tops. Further increase of the coupling strength will make two coupled kicked tops very difficultly evolve into the stationary state even with a very large imaginary part of $k$. Their entanglement becomes stationary after at least $10^{5}$ kicking steps. While for the case with $\operatorname{Re}(k)=0.25$, the imaginary part of $k$ heavily affects the stationary entanglement, and the results were shown in Fig.6(b). Similarly, the stationary log-negativity has its local maximum at certain value of $\varepsilon$ between 0.7 and 0.8 , and declines to 1 .

Similar to the previous results that chaotic (regular) semiclassical dynamical behaviors have connections with large (small) entanglement production in their corresponding quantum systems in Ref. [3 6, 20], from the Fig.2 and Fig.5, it can be found the larger (smaller) stationary log-negativity, the more chaotic (regular) dynamical behaviors of the log-negativity in the present quantum system with only total 9-dimensional Hilbert space. Moreover, comparing Fig.3(a) and Fig.5, the antimonotonic relation between the rank correlation coefficient and the stationary entanglement can be found, in which the mismatch over a short range between $k=4.5$ and $k=6$ could be conjecturally removed via taking average over a large number of initial states. Thus it is proposed to use this stationary log-negativity or $\eta_{d}$ defined in Eq.(1) as a candidate of the signature of quantum chaos in deep quantum regime. Obviously, the strength of the imaginary part $\operatorname{Im}(k)$ will affect the dynamical behaviors of the log-negativity. If the stationary log-negativity was adopted as the signature to investigate the border between the regular and chaotic regions in the stroboscopic quantum phase space or parameter space, it is suggested to choose a small enough imaginary part of $k$, i.e. $\operatorname{Im}(k) \ll \operatorname{Re}(k)$ to avoid significant disturbance to the system of interest.

In Fig.2, it can be observed the log-negativity of two tops achieves a local maximal value near $t=70$ for different values of the parameters $k$ and $p$. We have investigated the dependence of the local maximal log-negativity on the parameter $p$ for different values of $k$ and a fixed

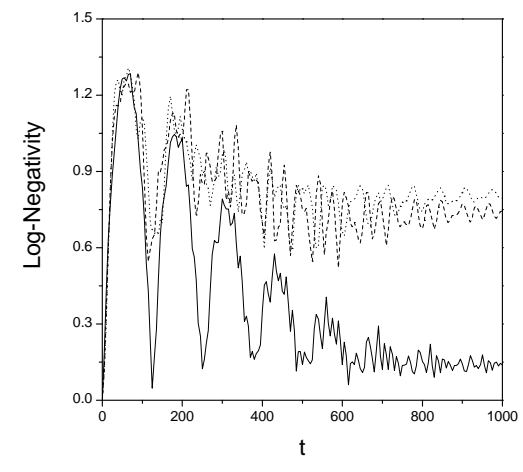

FIG. 4: The log-negativity of two tops is plotted as the function of $t$ for different nonlinear coefficients and pure initial spin coherent state. $j=1 ; \varepsilon=0.05 ; \gamma_{1}=-3 ; \gamma_{2}=3$; $p=0.0 ; \operatorname{Im}(k)=0.01 ;$ (Solid line) $\operatorname{Re}(k)=3.0 ;$ (Dash line) $\operatorname{Re}(k)=6.0 ;$ (Dot line) $\operatorname{Re}(k)=0.25$.

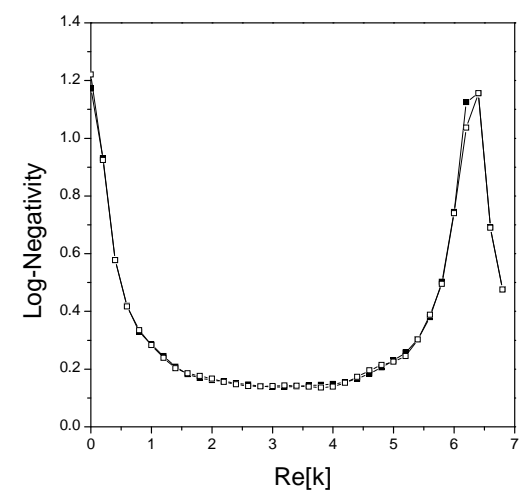

FIG. 5: The log-negativity of two tops at the kick step $t=1000$ is plotted as the function of the real part $\operatorname{Re}(k)$ of $k$ for two different pure initial spin coherent state. $j=1$; $\varepsilon=0.05 ; p=0 ; \operatorname{Im}(k)=0.01 ;$ (hole square) $\gamma_{1}=\gamma_{2}=$ $\exp (0.63 i) \tan (0.89 / 2)$, a chaotic point in phase space of the single kicked top with $k=3$ in Ref. [8]; (Real square) $\gamma_{1}=-3$; $\gamma_{2}=3$.

value of $\varepsilon=0.05$. They decline with $p$ at the slope of about 0.9 for the case with $\gamma_{1}=-3$ and $\gamma_{2}=3$. The slopes only slightly depend on the parameter $k$, but heavily correlate with the initial points labeled by $\gamma_{1}$ and $\gamma_{2}$. In the cases of $\gamma_{1}=-\gamma_{2}$, it is found the slopes decrease with the increase of absolute value of $\gamma_{1}$ due to the mixedness of the initial state decreases with $\left|\gamma_{1}\right| \geq 1$ for $p \in(0,0.5]$. For those kind of initial states in Eqs.(7$8)$, there may be quantum correlation between two tops though they are separable [29, 30]. Their quantum discord is dependent of the overlap $\left\langle\gamma_{1} \mid \gamma_{2}\right\rangle$ and $p$. The intermediate value of the overlap and $p$ could make two tops achieve the larger quantum discord. It is also interesting to investigate how the quantum discord and classical correlation evolve in the kicked coupled tops, which maybe embody good quantum-classical correspondence [31 34].

At last but not least, we should investigate the fidelity 

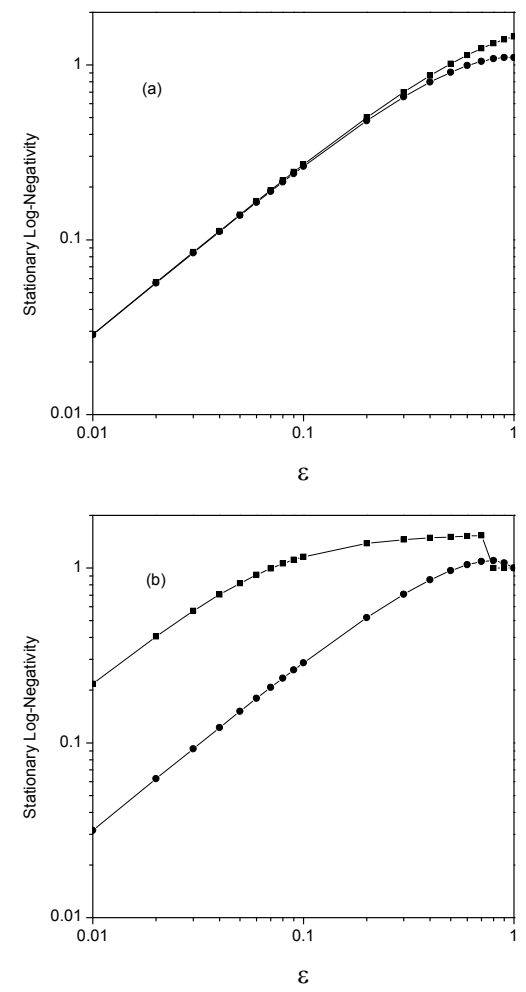

FIG. 6: The stationary log-negativity of two tops is plotted as the function of the coupling strength $\varepsilon$. $j=1$. (a) $\operatorname{Re}(k)=3 ;$ (b) $\operatorname{Re}(k)=0.25$. (Square) $\operatorname{Im}(k)=0.01$; (Circle) $\operatorname{Im}(k)=3.0$. In the case with $j=1$, it has been verified the stationary log-negativity is initial-state independent for both pure or mixed initial states in Eq.(7). In the case with $\operatorname{Re}(k)=3, \mathcal{N}(t \rightarrow \infty) \propto \varepsilon^{0.97}$ as $\varepsilon \leq 0.1$.

decay in the coupled kicked tops. Usually, the fidelity for two mixed states $\rho_{1}$ and $\rho_{2}$ is defined by [35]

$$
F\left(\rho_{1}, \rho_{2}\right)=\left[\operatorname{Tr}\left(\sqrt{\sqrt{\rho_{1}} \rho_{2} \sqrt{\rho_{1}}}\right)\right]^{2}
$$

Here, we adopt this fidelity to study the sensitivity of evolving state in the coupled kicked tops to the small perturbation, i.e. $\rho_{1}(t)$ and $\rho_{2}(t)$ represent two evolving density matrix of the kicked coupled tops with two slight different nonlinear coefficients $k$ and $k^{\prime}=k+\delta k(\delta k \ll 1)$, respectively. Initially $\rho_{1}(0)=\rho_{2}(0)$ belong to those class of states in Eqs.(7-8). Generally, the fidelity decay should be taken average of the overall initial states in the phase space, and the average fidelity decay rate can be used to build relations with the signatures of its classical chaotic counterpart such as the mean Lyapunov exponent 36 [38, 40]. The average fidelity decay typically exhibits three consecutive stages, i.e. the short-time parabolic decay, the followed intermediate-time asymptotic decay, and long-time saturation [37-39]. Nevertheless, it is still worthy to analyze the corresponding fidelity decay for some specific evolving states with manifested chaotic dynamical behaviors of entanglement. The key parameters in Fig.(2a) are adopted to reveal how the fidelity
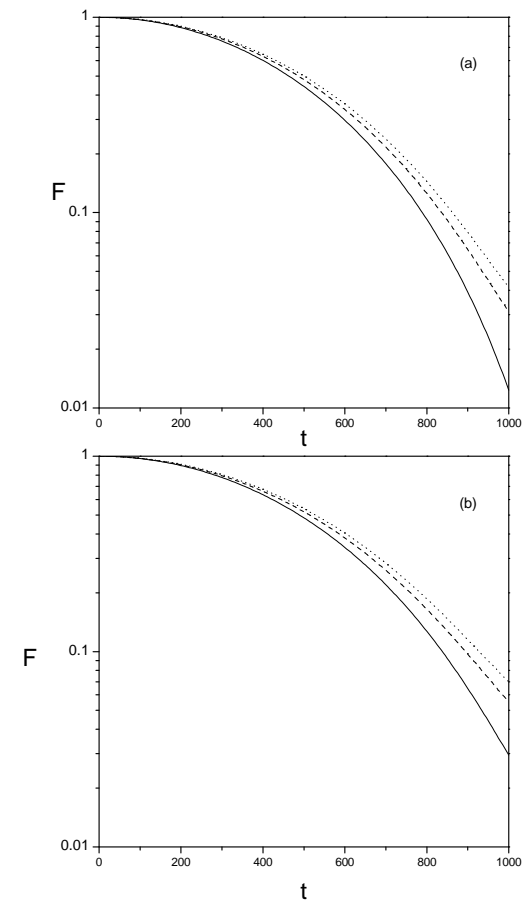

FIG. 7: The fidelity of the system is plotted as the function of $t$ with $j=1, k=0.25, k^{\prime}=0.26, \gamma_{1}=-3, \gamma_{2}=3$. (a) $\varepsilon / j=0.02$, (Solid line) $p=0 ;$ (Dash line) $p=0.2$; (Dot line) $p=0.5$. (b) $\varepsilon / j=0.05$, (Solid line) $p=0$; (Dash line) $p=0.2 ;$ (Dot line) $p=0.5$.
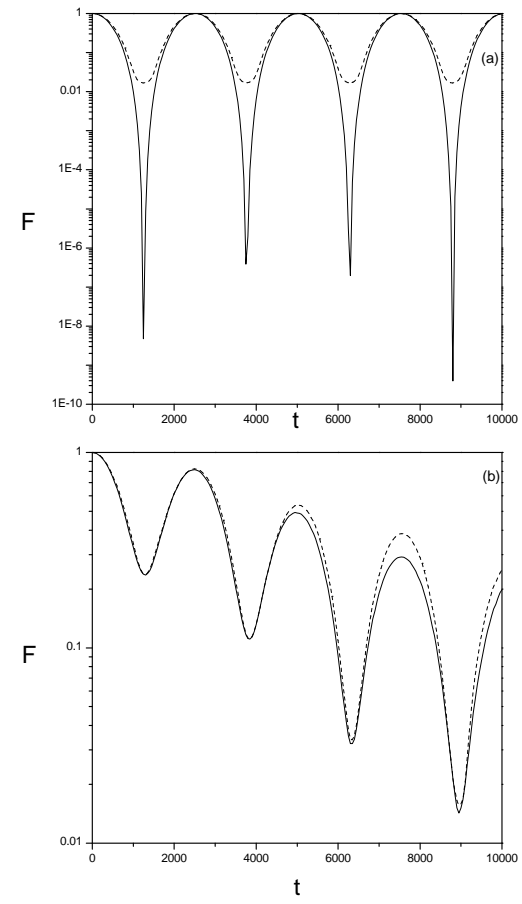

FIG. 8: The fidelity of the system is plotted as the function of $t$ with $j=1, k=0.25, k^{\prime}=0.26, \gamma_{1}=-3, \gamma_{2}=3$. (a) $\varepsilon / j=0$, (Solid line) $p=0$; (Dash line) $p=0.5$. (b) $\varepsilon / j=0.5$, (Solid line) $p=0 ;$ (Dash line) $p=0.5$. 
decays in such a case with chaotic dynamical behavior of bipartite entanglement. The results are displayed in Figs. (7-8). In the first stage, the fidelity more rapidly decays than exponential decay, and the decay rate decreases with the increase of mixedness and the coupling coefficient $\varepsilon$. Then, the fidelity revivals. When $\varepsilon=0$, it can be observed perfect recurrence of the fidelity, which implies the uncoupled kicked top with $k=0.25$ is regular in the long-time scale. In the strong coupling case with $\varepsilon=0.5$, initially the two trajectories of fidelity decay seem coincident, but after some time, the divergence is obvious and enlarged. In the kicked coupled spin-1, the coupling coefficient $\varepsilon$ plays the more crucial role in the chaotic dynamical behaviors of the entanglement than the nonlinear coefficient $k$, which may be the partial reason why the classical chaotic initial states of the individual top induces the more synchronous dynamical behaviors of entanglement than the regular initial states. Via replacing the operators of the spin $j=1$ by $\hat{J}_{z}^{(i)}=\sum_{n=1}^{2} \sigma_{z_{n}}^{(i)} / 2$ and $\hat{J}_{y}^{(i)}=\sum_{n=1}^{2} \sigma_{y_{n}}^{(i)} / 2$, where $\sigma_{z_{n}}^{(i)}$ and $\sigma_{y_{n}}^{(i)}$ are the Pauli operators of the qubit with label $\{i=1,2 ; n=1,2\}$, the kicked coupled spin 1 is equivalent to a kicked four-qubit 2D Ising square. Four-qubit 2D Ising square may be one of the basic cells for constructing the universal quantum gate or measurement-based quantum computation [41]. Therefore, to fully investigate the border between the regular and chaotic regions both in the quantum phase space or parameter space is very important and desirable in the future work.

In summary, this Letter deals with the dynamical pattern of entanglement in the simplest kicked coupled symmetric tops with $j=1$. The main result is to preliminarily reveal the potential antimonotonic relation between the scaled rank correlation coefficient $\eta_{d}$ defined in Eq.(1) and the stationary entanglement induced by suitable nonhermitian term in the coupled kicked tops. However, further calculations including large number of initial states and key parameters in the system of the coupled kicked tops are necessary to finally qualify these signature candidates of quantum chaos in low dimensional Hilbert space. Meanwhile, there is still lack of a reasonable physical interpretation why the rank correlation coefficient has such an antimonotonic relation with the stationary entanglement in the system of amplified kicked coupled spin-1.

This work was supported in part by National 973 Program of China (Grant No. 2013CB329201).
[1] W.H. Zurek, Phys.Rev. D 24, 1516 (1981); 26, 1862 (1982); W.H. Zurek, Rev. Mod. Phys. 75, 715 (2003).

[2] F. Haake, M. Kus, and R. Scharf, Z. Phys. B 65, 381 (1987); F. Haake, Quantum Signatures of Chaos, 2nd ed. (Springer-Verlag, Berlin, 2000)

[3] S. Chaudhury, A. Smith, B.E. Anderson, S. Ghose, and P.S. Jessen, Nature 461, 768 (2009).

[4] X. Wang, S. Ghose, B.C. Sanders, and B. Hu, Phys. Rev. E 70, 016217 (2004).

[5] S. Ghose, and B.C. Sanders, Phys. Rev. A 70, 062315 (2004).

[6] S. Ghose, R. Stock, P. Jessen, R. Lal, and A. Silberfarb, Phys. Rev. A 78, 042318 (2008).

[7] K. Furuya, M.C. Nemes, and G.Q. Pellegrino, Phys. Rev. Lett. 80, 5524 (1998).

[8] P.A. Miller and S. Sarkar, Phys. Rev. E 60, 1542 (1999); P.A. Miller and S. Sarkar, Nonlinearity 12, 419 (1999); S. Nag, A. Lahiri, and G. Ghosh, Phys. Lett. 292A, 43 (2001).

[9] A. Lakshminarayan, Phys. Rev. E 64, 036207 (2001).

[10] J.N. Bandyopadhyay and A. Lakshminarayan, Phys. Rev. Lett. 89, 060402 (2002); ibid. Phys. Rev. E 69, 016201 (2004).

[11] A. Tanaka, H. Fujisaki, and T. Miyadera, Phys.Rev. E 66 045201(R) 2002); H. Fujisaki, T. Miyadera, and A. Tanaka, Phys. Rev. E 67, 066201 (2003); H. Fujisaki, A. Tanaka and T. Miyadera, J. Phys. Soc. Jpn. 72, 111 (2003).

[12] S.-B. Li and J.-B. Xu, Phys. Rev. E 74, 046204 (2006).

[13] R.F. Abreu and R.O. Vallejos, Phys. Rev. A 73, 052327 (2006).

[14] B. Georgeot and D.L. Shepelyansky, Phys. Rev. E 62, 3504 (2000); 62, 6366 (2000).

[15] B. Georgeot and D.L. Shepelyansky, Phys. Rev. Lett.86,
2890(2001).

[16] P.G. Silvestrov, H. Schomerus, and C.W.J. Beenakker, Phys. Rev. Lett. 86, 5192 (2001).

[17] S. Bettelli and D.L. Shepelyansky, Phys. Rev. A 67, 054303 (2003).

[18] R.M. Angelo, K. Furuya, M.C. Nemes, and G.Q. Pellegrino, Phys. Rev. E 60, 5407 (1999).

[19] C.M. Trail, V. Madhok, I.H. Deutsch, Phys. Rev. E 78, 046211 (2008).

[20] R. Demkowicz-Dobrzański, M. Kuś, Phys. Rev. E 70, 066216 (2004); ibid. 78, 019904 (2008).

[21] N.N. Chung and L.Y. Chew, Phys. Rev. E 80, 016204 (2009).

[22] H. Kubotani, S. Adachi, and M. Toda, Phys. Rev. Lett. 100, 240501 (2008).

[23] M. Lombardi and A. Matzkin, Phys. Rev. A 73, 062335 (2006).

[24] Y.S. Weinstein and C.S. Hellberg, Phys. Rev. Lett. 95, 030501 (2005).

[25] S. Habib, K. Shizume, and W.H. Zurek, Phys. Rev. Lett. 80, 4361 (1998).

[26] M.G. Kendall, Rank Correlation Methods, 4th ed. (Griffin., London, 1970).

[27] G. Vidal, R.F. Werner, Phys. Rev. A 65, 032314 (2002); M.B. Plenio, Phys. Rev. Lett. 95, 090503 (2005).

[28] G.M. D'Ariano, L.R. Evangelista, and M. Saraceno, Phys. Rev. A 45, 3646 (1992).

[29] H. Ollivier and W.H. Zurek, Phys. Rev. Lett. 88, 017901 (2001).

[30] L. Henderson and V. Vedral, J. Phys. A 34, 6899 (2001).

[31] W.H. Zurek, Los Alamos Sci. 27,2 (2002).

[32] S. Haroche, Phys. Today 51, 36 (1998).

[33] W.-G. Wang, J. Gong, G. Casati, and B. Li, Phys. Rev. A 77, 012108 (2008). 
[34] D. Monteoliva and J.P. Paz, Phys. Rev. Lett. 85, 3373 (2000); Phys. Rev. E 64, 056238 (2001).

[35] R. Jozsa, J. Mod. Opt. 41, 2315 (1994).

[36] A. Peres, Phys. Rev. A 30, 1610 (1984).

[37] T. Gorin, T. Prosen, T.H. Seligman, and M. Žnidarič, Phys. Rep. 435, 33 (2006), and references therein.

[38] Ph. Jacquod, P.G. Silvestrov, and C.W.J. Beenakker, Phys. Rev. E 64, 055203(R) (2001).

[39] R.A. Jalabert and H.M. Pastawski, Phys. Rev. Lett. 86,
2490 (2001).

[40] J. Emerson, Y.S. Weinstein, S. Lloyd, and D.G. Cory, Phys. Rev. Lett. 89, 284102 (2002).

[41] M. Van den Nest, W. Duer, H.J. Briegel, Phys. Rev. Lett. 100, 110501 (2008); H.J. Briegel, D.E. Browne, W. Dür, R. Raussendorf, and M. Van den Nest, Nature Phys. 5, 19 (2009); J.A. Jones, Phys. Rev. A 67, 012317 (2003). 